\title{
Gradient Kähler-Ricci Solitons and Periodic Orbits
}

\author{
Huai-Dong CaO and Richard S. Hamilton
}

\begin{abstract}
We study Hamiltonian dynamics of gradient Kähler-Ricci solitons that arise as limits of dilations of singularities of the Ricci flow on compact Kähler manifolds. Our main result is that the underlying spaces of such gradient solitons must be Stein manifolds. Moreover, on all most all energy surfaces of the potential function $f$ of such a soliton, the Hamiltonian vector field $V_{f}$ of $f$, with respect to the Kähler form of the gradient soliton metric, admits a periodic orbit. The latter should be of significance in the study of singularities of the Ricci flow on compact Kähler manifolds in light of the "little loop lemma" principle in [10].
\end{abstract}

\section{Introduction.}

Let $X$ be a noncompact complex manifold. Consider the Ricci flow

$$
\frac{\partial}{\partial t} g_{i \bar{j}}=-R_{i \bar{j}}
$$

which evolves a complete Kähler metric $g_{i \bar{j}}$ on $X$ by its Ricci tensor $R_{i \bar{j}}$. A solution metric $g_{i \bar{j}}$ of Eq.(1) is called a gradient Kähler-Ricci soliton if it moves along Eq.(1) under a one-parameter family of biholomorphisms of $X$ generated by a holomorphic gradient vector field. Namely, $g_{i \bar{j}}(t)=\phi^{*}(t) g_{i \bar{j}}(0)$, where $\phi(t)$ is a 1-parameter family of automorphisms of $X$ generated by some holomorphic gradient vector field. More precisely, this means that the Ricci tensor of the metric $g_{i j}$ can be expressed as

$$
R_{i \bar{j}}=f_{i \bar{j}}, \quad \text { with } \quad f_{, i j}=0
$$

for some real-valued function $f$ on $X$. Note that the condition $f_{, i j}=0$ is equivalent to saying that the gradient vector field $\nabla f$ is holomorphic.

Understanding gradient Ricci solitons is very important in the study of Ricci flow, since they often arise as limits of dilations of singularities of the

The authors' research was supported in part by NSF. 
Ricci flow. This was first observed by the second author [10]. For example, consider the normalized Ricci flow

$$
\frac{\partial}{\partial t} g_{i \bar{j}}=-R_{i \bar{j}}+g_{i \bar{j}}
$$

on a $n$-dimensional compact Kähler manifold $M^{n}$ with positive first Chern class $C_{1}(M)$. It is known ([1], [2]) that the solution to Eq.(3) exists for all time $0 \leq t<\infty$. A key problem in the study of asymptotic behavior of solutions to Eq.(3) is to obtain a uniform estimate of the evolved curvature tensor. By singularities of the Ricci flow (3) we mean solutions to Eq.(3) with unbounded curvature as time $t$ tends to the infinity. It is conjectured that singularities of Eq.(3) do not occur and solutions to Eq.(3) either converges to a Kähler-Einstein metric or a homothetically shrinking gradient Kähler-Ricci soliton of Eq.(3), a solution moving along Eq.(3) under a one-parameter family of biholomorphisms of $X$ generated by a holomorphic gradient vector field. On the other hand, if singularities do occur then, as indicated in [10], certain limit of parabolic dilations, dilating time as distance squared, of the solution metric $g_{i \bar{j}}(t)$ to Eq.(3) is a complete solution metric to Eq.(1) on a noncompact complex manifold $X$ defined for $-\infty<t<\infty$ with uniformly bounded curvature. When the holomorphic bisectional curvature of the initial metric is positive this limit metric has nonnegative holomorphic bisectional curvature where the scalar curvature $R$ assumes its maximum in space-time. It is shown in [4] that such a solution metric $g_{i \bar{j}}$ is necessarily a gradient Kähler-Ricci soliton. Thus singularities of the Ricci flow (3) are related to these special gradient Kähler-Ricci solitons. So far, the only known examples of gradient Kähler-Ricci solitons are the rotationally symmetric ones. They are the "cigar" soliton on the complex plane found by the second author [6] and its higher dimensional analogues on $\mathbf{C}^{n}$ found by the first author [3]. Not much was known about general structures of gradient solitons. In this paper, we explore the structure of gradient solitons and the symplectic geometry of periodic orbits of certain special Hamiltonian functions on gradient Kähler-Ricci solitons, which arise as limits of dilations of singularities of the Ricci flow (3)). Our main result can be stated as follows.

Theorem 1.1. Let $g_{i \bar{j}}$ be a complete gradient Kähler-Ricci soliton on a noncompact complex manifold $X$ with positive Ricci curvature such that the scalar curvature $R$ assumes its maximum in space-time. Let $f$ be the potential function of the soliton as defined in (2). Then $f$ is a plurisubharmonic exhaustion function. Hence $X$ must be a Stein manifold. Furthermore, near 
each level surface $S_{c}=\{f=c\} \subset X$ there exists a periodic orbit for the Hamiltonian vector field $V_{f}$ of $f$ with respect to the symplectic form $\omega$ defined by the Kähler form of $g_{i \bar{j}}$.

Our work is motivated by the second author's "little loop lemma" principle [10], which roughly says that there should be no small loop in a big flat region for the solution of Ricci flow whenever Hanarck estimates ([7], [2]) hold. This turns out to be an effective tool to rule out singularities in some cases. Our work is also motivated by the examples of the cigar soliton and its higher dimensional versions, where periodic orbits of the corresponding Hamiltonian vector fields do exist and have uniformly finite length.

The existence of periodic orbits on a compact energy surface is an old question of the qualitative theory of Hamiltonian systems. We refer the reader to the book of Hofer and Zehnder [11] for history and recent development on this rich subject.

The proof of Theorem 1.1 is based on the structure equations of gradient Ricci solitons, the symplectic capacity theory of Hofer-Zehnder (see [11]), and a result of Eliashberg-Gromov [5]. One interesting feature is that we use both the Kähler form $\omega$ and the Ricci form $\rho=\sqrt{-1} \partial \bar{\partial} f$ of the soliton metric as symplectic forms on $X$. On one hand, we need to use $\omega$ as the symplectic form due to geometric considerations. On the other hand, the Ricci form $\rho$ is more naturally adapted to symplectic embedding problems. It turns out that $X$, with the Ricci form $\rho$ as the symplectic form, can be symplectically embedded into $\mathbb{R}^{2 n}$ with the standard symplectic form. Moreover, based on a result of Eliashberg-Gromov in [5], we are able to show that there exists a symplectic embedding of a neighborhood of the level surface $S_{c}$, with symplectic form $\omega$, into the symplectic manifold $(X, \rho)$. It then follows from the capacity theory of Hofer-Zehnder [11] that level surface $S_{c}$ has a periodic orbits for almost all $c>0$.

Acknowledgement. The first author would like to thank Yasha Eliashberg for valuable suggestions and Viktor Ginzburg for helpful discussions.

\section{Gradient Kähler-Ricci solitons.}

In this section we discuss various aspects of gradient Kähler-Ricci solitons. First, we derive the first order and the second order equations satisfied by gradient Ricci solitons. To certain extent, these equations characterize gradient Kähler-Ricci solitons (see [4]). Next, we describe the rotatinally symmetric examples, namely, the cigar soliton on $\mathbf{C}$ and its analogues on $\mathbf{C}^{n}$. 


\subsection{Ricci soliton equations.}

Let $g_{i \bar{j}}$ be a gradient Kähler-Ricci soliton of Eq.(1) so that it satisfies Eq.(2). From the commutation formulas for the covariant derivatives we have

$$
f_{, i k \bar{j}}-f_{, i \bar{j} k}=R_{i \bar{j} k \bar{q}} f_{, q} .
$$

On the other hand, taking covariant derivate of the first equation in (2) and using the second equation, we have

$$
f_{, i k \bar{j}}-f_{, i \bar{j} k}=-R_{i \bar{j}, k} .
$$

Hence we get

$$
R_{i \bar{j}, k}+R_{i \bar{j} k \bar{q}} f_{, q}=0 .
$$

Taking the trace, we get

$$
R_{, k}+R_{k \bar{l}} f_{, l}=0 .
$$

Differentiating (4) once more we obtain

$$
R_{i \bar{j}, k \bar{l}}+R_{i \bar{j} k \bar{q}, \bar{l}} f_{, q}+R_{i \bar{j} k \bar{q}, f_{, q \bar{l}}}=0 .
$$

By the second Bianchi identity and (2) we have

$$
R_{i \bar{j}, k \bar{l}}+R_{i \bar{j} k \bar{l}, \bar{q}} f_{, q}+R_{i \bar{j} k \bar{q}} R_{q \bar{l}}=0 .
$$

Taking the trace in the above equation, we obtain

$$
\Delta R_{i \bar{j}}+R_{i \bar{j} k \bar{l}} R_{l \bar{k}}+R_{i \bar{j}, \bar{k}} f_{, k}=0 .
$$

Equations (4) and (6) are the first order and the second order equations satisfied by gradient Kähler-Ricci solitons. They are also related to the $(1,1)$ tensor in the Hanarck estimates (see [2]).

\subsection{Rotationally symmetric examples.}

In [6], the second author wrote down the first example of a gradient Ricci soliton, called the cigar soliton, on the complex plane $\mathbf{C}$. It has the form

$$
d s^{2}=\frac{|d z|^{2}}{1+|z|^{2}}, \quad z \in \mathbf{C} .
$$


Its potential function $f$ is given by $f=\log \left(1+|z|^{2}\right)$ and its scalar curvature is given by

$$
R=\frac{1}{1+|z|^{2}}
$$

It is easy to verify that $\nabla f=z$. Here $\nabla f$ denotes the gradient of $f$ with respect to the soliton metric $d s^{2}$. So the cigar soliton flows towards the origin by conformal dilations and has positive Gaussian curvature. It is asymptotic to a flat cylinder at infinity and has maximal scalar curvature 1 at the origin, hence the name cigar soliton.

In [3], the first author studied the existence of rotationally symmetric gradient Kähler-Ricci solitons and proved the following

Proposition 2.1. For $n \geq 1$, there exists on $\mathbf{C}^{n}$ a complete rotationally symmetric gradient Kähler-Ricci soliton of positive sectional curvature.

Our rotationally symmetric solitons are constructed by considering $U(n)$ invariant Kähler metrics of the form

$$
g_{i \bar{j}}=\partial_{i} \partial_{\bar{j}} \Phi(z, \bar{z}),
$$

with the Kähler potential $\Phi(z, \bar{z})$ expressed as

$$
\Phi(z, \bar{z})=u(t), \quad\left(t=\log |z|^{2}\right) .
$$

Here $u(t)$ is a smooth function defined on $(-\infty, \infty)$ satisfying the differential inequalities

$$
u^{\prime}(t)>0, \quad u^{\prime \prime}(t)>0, \quad t \in(-\infty, \infty)
$$

and as $t \rightarrow-\infty$, has an expansion

$$
u(t)=a_{0}+a_{1} e^{t}+a_{2} e^{2 t}+\cdots . \quad\left(a_{1}>0\right)
$$

Note that the metric $g_{i \bar{j}}$ is given by

$$
g_{i \bar{j}}=\partial_{i} \partial_{\bar{j}} u(t)=e^{-t} u^{\prime}(t) \delta_{i \bar{j}}+e^{-2 t} \bar{z}_{i} z_{j}\left(u^{\prime \prime}(t)-u^{\prime}(t)\right) .
$$

It is natural to consider

$$
f(t)=-\log \operatorname{det}\left(g_{i \bar{j}}\right)=n t-(n-1) \log u^{\prime}(t)-\log u^{\prime \prime}(t)
$$

so that the Ricci tensor of the metric $g_{i \bar{j}}$ is given by $R_{i \bar{j}}=\partial_{i} \partial_{\bar{j}} f(t)$. 
According to (2), the metric $g_{i \bar{j}}$ will be a gradient Kähler-Ricci soliton with potential function $f$ if the gradient vector field $V=\nabla f$ is holomorphic. By a direct computation,

$$
V^{i}=g^{i \bar{j}} f_{, \bar{j}}=z_{i} \frac{f^{\prime}(t)}{u^{\prime \prime}(t)} .
$$

Hence $V$ is holomorphic if and only if $f^{\prime}(t)=\alpha u^{\prime \prime}(t)$ for some constant $\alpha \in \mathbb{R}$. By setting $\phi(t)=u^{\prime}(t)$ and by appropriate scaling (in metric) and dilation (in $z$ ), the equation satisfied by a gradient soliton becomes

$$
\phi^{n-1} \phi^{\prime} e^{\phi}=e^{n t} .
$$

An implicit solution of $\phi$ is given by

$$
\sum_{k=0}^{n-1}(-1)^{n-k-1} \frac{n !}{k !} \phi^{k} e^{\phi}=e^{n t}+(-1)^{n-1} n !
$$

It is easy to check from (10) that the solution $\phi=u^{\prime}$ satisfies the required asymptotic condition (8) and the differential inequalities (7). Thus it gives rise to a $U(n)$-invariant gradient Kähler-Ricci soliton metric $g$ on $\mathbf{C}^{n}$. Furthermore, the sectional curvature of the soliton metric turns out to be positive (see [3]). Note that when $n=1$, we have $\phi=\log \left(1+e^{t}\right)$ and the soliton metric is nothing but the cigar soliton.

From (10) or (9), it is also easy to see that

$$
\lim _{t \rightarrow \infty} t^{-1} \phi(t)=n, \quad \lim _{t \rightarrow \infty} \phi^{\prime}(t)=n
$$

which in turn imply that the soliton metric $g$ is complete. Let $s$ denote the distance function from the origin with respect to $g$. Then it can be shown that the soliton metric $g$ on $\mathbf{C}^{n}(n \geq 2)$ satisfies the following two properties: (1) The volume of the geodesic ball $B_{g}(0, s)$ with respect to the metric $g$ grows like $s^{n} ;(2)$ The scalar curvature $R$ of the metric $g$ decays like $1 / s$.

Finally, we point out that on this Ricci soliton the sphere $S^{2 n-1}$ at radius $s$ looks like an $S^{1}$ bundle over $C P^{n-1}$ where the $C P^{n-1}$ has diameter on the order of $\sqrt{s}$ while the $S^{1}$ fiber has diameter on the order of 1 . This $S^{1}$ fiber is nothing but a periodic orbit for the Hamiltonian vector field $V_{f}=J \nabla f$, $J$ being the standard complex structure on $\mathbf{C}^{n}$, of $f$. For the cigar soliton these periodic orbits are simply given by parallel circles, whose lengths are bounded by $2 \pi r / \sqrt{1+r^{2}}$. Here $r$ denotes the Euclidean radii of the circles. 


\section{Symplectic capacity theory of Hofer-Zehnder.}

In this section, we recall the symplectic capacity function introduced by Hofer and Zehnder (see [11]) and the related existence theorem of periodic orbits on energy surfaces. The results will be used in the next section to prove Theorem 1.1.

\subsection{Definition of the capacity $c_{0}$.}

Consider the class of all symplectic manifolds $(M, \omega)$ possibly with boundary and of fixed dimension $2 n$. A symplectic capacity is a map $(M, \omega) \rightarrow c(M, \omega)$ which associates with every symplectic manifold $(M, \omega)$ a nonnegative number or $\infty$, satisfying the following three properties.

(1) Monotonicity: $c(M, \omega) \leq c(N, \tau)$ if there exists a symplectic embed$\operatorname{ding} \phi:(M, \omega) \rightarrow(N, \tau)$.

(2) Conformality: $c(M, a \omega)=|a|(M, \omega)$ for all $a \in \mathbb{R}, a \neq 0$.

(3) Nontriviality: $c\left(B_{1}, \omega\right)=\pi=\left(Z_{1}, \omega_{0}\right)$ for the open unit $B_{1}$ and the open symplectic cylinder $Z_{1}=\left\{(x, y) \in \mathbb{R}^{2 n}: x_{1}^{2}+y_{1}^{2}<1\right\}$ in the standard symplectic vector space $\left(\mathbb{R}^{2 n}, \omega_{0}\right)$.

It follows from the conformality property and nontriviality property of a symplectic capacity that for the open ball of radius $r>0$ in $\mathbb{R}^{2 n}, c(B(r))=$ $\pi r^{2}$. In particular, the capacity $c(K)$ of every compact subset $K$ in $\mathbb{R}^{2 n}$ is finite.

Hofer and Zehnder [11] introduced a distinguished capacity function $c_{0}$. The capacity $c_{0}(M, \omega)$ measures the minimal $C^{0}$-oscillation of special Hamiltonian functions $H: M \rightarrow \mathbb{R}$, needed in order to conclude the existence of a distinguished periodic orbit having small period.

Now let's recall the definition of the capacity $c_{0}$. On a symplectic manifold $(M, \omega)$, to a smooth function $H: M \rightarrow \mathbb{R}$ there belongs a unique Hamiltonian vector field $V_{H}$ on $M$ defined by

$$
\left(i_{V_{H}} \omega\right)(x)=\omega\left(V_{H}(x), w\right)=-d H(x)(w), \text { for } w \in T_{x}(M)
$$

and $x \in M$. With respect to a given Riemannian metric $\langle$,$\rangle on M$ and a compatible almost complex structure $J$, the Hamiltonian vector field is represented by

$$
V_{H}(x)=J \nabla H(x) \in T_{x} M
$$


where $\nabla H$ is the gradient of $H$ with respect to the metric $\langle$,$\rangle . A T$-periodic solution $x(t)$ of the Hamiltonian equation

$$
x^{\prime}=V_{H}(x) \text { on } M
$$

is a solution satisfying the boundary conditions $x(T)=x(0)$ for some $T>0$. Denote by $\mathcal{H}(M, \omega)$ the set of smooth functions $H$ on $M$ satisfying the following properties:

(a) There is a compact set $K \subset(M \backslash \partial M)$ (depending on $H$ ) such that $H(x) \equiv m(H)$, a positive constant, on $M \backslash K$.

(b) There is an open set $U \subset M$ (depending on $H$ ) on which $H \equiv 0$.

(c) $0 \leq H(x) \leq m(H)$ for all $x \in M$.

(d) All the periodic solutions of Eq. (11) on $M$ are either constant or have a period $T>1$.

Hofer and Zehnder [11] defined

$$
c_{0}(M, \omega)=\sup \{m(H): H \in \mathcal{H}(M, \omega)\}
$$

and proved

Theorem 3.1. The function $c_{0}$ is a symplectic capacity.

\subsection{A existence theorem of periodic orbits.}

The flow $\phi^{t}$ of a Hamiltonian vector field

$$
x^{\prime}=V_{H}(x), x \in M
$$

leaves the level sets of the function $H$ on $M$ invariant. Fixing a value $c$ of this energy function which we can assume to be $c=1$, suppose that the energy surface

$$
S=\{x \in M: H(x)=1\}
$$

is compact and regular, i.e., $d H(x) \neq 0$, for $x \in S$. Then $S \subset M$ is a smooth and compact submanifold of codimension 1 whose tangent space at $x \in S$ is given by

$$
T_{x} S=\left\{\xi \in T_{x} M: d H(x) \xi=0\right\} .
$$


By the definition of a Hamiltonian vector field we have $V_{H}(x) \in T_{x} S$ so that $V_{H}$ is a nonvanishing vector field on $S$ whose flow exists for all time since $S$ is compact. On the other hand, since $S$ is compact and regular there is an open and bounded neighborhood $U$ of $S$ which is filled with compact and regular energy surfaces having energy values near $c=1$ :

$$
U=\bigcup_{\lambda \in I} S_{\lambda}
$$

where $I$ is an open interval around $\lambda=1$ and $S_{\lambda}=\{x \in U: H(x)=\lambda\}$ is diffeomorphic to the given surface $S=S_{1}$.

Now we can state a result of Hofer and Zehnder ([11], p. 106) about the existence of periodic orbits.

Theorem 3.2. Let $S$ be a compact regular energy surface for the Hamiltonian vector field $V_{H}$ on $(M, \omega)$. Assume there is an open neighborhood $U$ of $S$ having finite capacity: $c_{0}(U, \omega)<\infty$. Then there is a dense subset $\Sigma \subset I$ such that for $\lambda \in \Sigma$ the energy surface $S_{\lambda}$ has a periodic solution of $V_{H}$.

\section{The Proof of Theorem 1.1.}

Let $g_{i \bar{j}}$ be a complete gradient Kähler-Ricci soliton on $X$ with positive Ricci curvature such that the scalar curvature $R$ assumes its maximum $R_{\max }=1$ at the point $O$, called the origin of $X$. Let $f$ be the potential function of the gradient soliton $g_{i \bar{j}}$ defined in Eq.(2).

Lemma 4.1. Let $g_{i \bar{j}}$ be a complete gradient Kähler-Ricci soliton on $X$. Then we have

$$
R+|\nabla f|^{2}=C
$$

on $X$, where $C$ is a positive constant.

Proof. Differentiating $R+|\nabla f|^{2}$ and using (2) and (5), we obtain

$$
\left(R+|\nabla f|^{2}\right)_{i}=R_{, i}+f_{j} f_{i \bar{j}}=R_{, i}+f_{j} R_{i \bar{j}}=0 .
$$

Hence $R+|\nabla f|^{2}$ is a constant.

Proposition 4.2. The potential function $f$ is a strictly convex exhaustion function on $X$ and assumes its minimum at the origin $O$. As a consequence, $X$ is necessarily a Stein manifold. 
Proof. The argument is basically the same as in [10]. Since the Ricci curvature $R_{i \bar{j}}$ is positive, it follows from the first equation in (2) that $f$ is plurisubharmonic. Furthermore, the second equation in (2) implies that $f$ is in fact strictly convex on $X$. Thus it remains to show that $f$ is proper, i.e., the set $\{f \leq c\}$ is compact for every positive number $c>0$, and assumes its minimum at $O$. From Lemma 4.1 we know that

$$
R+|\nabla f|^{2}=C
$$

for some positive constant $C$. Hence $|\nabla f|$ is smallest at the origin $O$, since $R$ has maximum at $O$. We claim that $C=1$. If not, then $C>1$ and $|\nabla f|^{2}>0$ everywhere. Consider a gradient path of $f$ through the origin and parametrized by $z^{i}=z^{i}(u)$ with $x^{i}$ at the origin at $u=0$ and

$$
\frac{d z^{i}}{d u}=g^{i \bar{j}} f_{\bar{j}} .
$$

Then

$$
\frac{d}{d u}|\nabla f|^{2}=2 g^{i \bar{l}} g^{k \bar{j}} R_{k \bar{l}} f_{\bar{j}}>0
$$

since $R_{i \bar{j}}>0$ and $|\nabla f|^{2}>0$. Then $|\nabla f|^{2}$ isn't smallest at the origin, a contradiction. Therefore $C=1$ and hence $\nabla f=0$ at the origin. Note also, from (12), that $|\nabla f|^{2}$ is bounded from above by 1 . These facts, together with the fact that $f$ is strictly convex, implies that $f$ is least at the origin and that $f$ is proper. Now a theorem of Grauert says that $X$ must be a Stein manifold.

Note that a plurisubharmonic function $\phi$ on a Stein manifold $M$ gives rise to a positive definite closed real $(1,1)$ form

$$
\omega_{\phi}=\sqrt{-1} \partial \bar{\partial} \phi
$$

and hence a symplectic form, on $M$.

Lemma 4.3. There exists a symplectic embedding $H:(X, \rho) \rightarrow\left(\mathbf{C}^{n}, \omega_{0}\right)$, where $\omega_{0}=\frac{\sqrt{-1}}{2} \sum_{i} d z^{i} \wedge d \bar{z}^{i}$ is the standard symplectic form on $\mathbf{C}^{n}$, and $\rho=\sqrt{-1} \partial \bar{\partial} f$ is the Ricci form of the soliton metric $g_{i \bar{j}}$.

Proof. We may write $\rho=d \eta$ and $\omega_{0}=d \eta_{0}$, where $\eta$ and $\eta_{0}$ are real 1forms on $X$ and $\mathbf{C}^{n}$ respectively. From Darboux's theorem, there exists a 
symplectic diffeomorphism $h:(U, \rho) \rightarrow\left(U_{0}, \omega_{0}\right)$ from a neighborhood $U$ of $O \in X$ onto a neighborhood $U_{0}$ of the origin in $\mathbf{C}^{\mathbf{n}}$. We denote

$$
\tilde{\eta}_{0}=\left(h^{-1}\right)^{*}(\eta)
$$

the push-forward of $\eta$. Then the form $\eta_{0}-\tilde{\eta}_{0}$ is closed on $U_{0}$. Hence $\eta_{0}-\tilde{\eta}_{0}=d \psi$ for some function $\psi$ in a smaller neighborhood $U_{0}^{\prime} \subset U_{0}$. Extend this function $\psi$, still denoted as $\psi$, to entire $\mathbf{C}^{n}$ so that $\psi \equiv 0$ outside $U_{0}$. Define the 1 -form

$$
\eta_{0}^{\prime}=\eta_{0}-d \psi
$$

Then $\eta_{0}^{\prime}=\eta_{0}$ outside $U_{0}, \eta_{0}^{\prime}=\tilde{\eta}_{0}$ on $U_{0}^{\prime}$ and

$$
d \eta_{0}^{\prime}=d \eta_{0}=\omega_{0}
$$

Let $W^{\prime}$ be the vector field, which is integrable, on $\mathbf{C}^{n}$ dual to $\eta_{0}^{\prime}$ with respect to $\omega_{0}$. Then, by an easy argument, we can obtain a unique extension $H$ : $(X, \rho) \rightarrow\left(\mathbf{C}^{n}, \omega_{0}\right)$ of $h:\left(h^{-1}\left(U_{0}^{\prime}\right), \rho\right) \rightarrow\left(U_{0}^{\prime}, \omega_{0}\right)$ by following the trajectries of $W=\nabla_{g_{f}} f$ and $W^{\prime}$ respectively. Here $\nabla_{g_{f}} f$ denotes the gradient vector field of $f$ with respect the Kähler metric defined by the Ricci form $\rho$.

Next we need the following result of Eliashberg-Gromov in [5].

Proposition 4.4. Let $\phi$ and $\psi$ be two exhaustion plurisubharmonic functions on a Stein manifold $M$. Then there exists a symplectic diffeomorphism $G:\left(M, \omega_{\phi}\right) \rightarrow\left(M, \omega_{\psi}\right)$.

Lemma 4.5. For every compact subset $K \subset X$, there exists a symplectic embedding $G:(K, \omega) \rightarrow(X, \rho)$.

Proof. For simplicity, we assume that $K=\{f \leq c\}$ for some constant $c>0$. Consider the compact set $K^{\prime}=\left\{f \leq c^{\prime}\right\}$ for some $c^{\prime}>c$ so that $K \subset K^{\prime}$. We can write the Kähler form $\omega=\sqrt{-1} \partial \bar{\partial} \phi$ for some real-valued function $\phi$ on $X$, since $\omega$ is a closed $(1,1)$ form and $X$ is simply connected. We may assume $\phi \geq 0$ on $K$. Choose a convex diffeomorphism $h: \mathbb{R} \rightarrow \mathbb{R}$ such that $\tilde{f}=h \circ f$ is also a plurisubharmonic exhaustion function on $X, \tilde{f}=f$ on $K$, and $\tilde{f}_{\mid \partial K^{\prime}}$ very large. Define

$$
\psi=\max (\tilde{f}-c, \phi)= \begin{cases}\phi & \text { on } K \\ \tilde{f}-c & \text { outside } K^{\prime}\end{cases}
$$


Then $\psi$ is another plurisubharmonic exhaustion function on $X$. By Proposition 4.4, there exists a symplectic diffeomorphism $G:\left(X, \omega_{\psi}\right) \rightarrow(X, \rho=$ $\left.\omega_{f}\right)$. But on $K$ we have $\omega_{\psi}=\sqrt{-1} \partial \bar{\partial} \phi=\omega$. Hence the restriction of $G$ to $K$ gives a symplectic embedding of $(K, \omega)$ into $(X, \rho)$.

Combining Lemma 4.3 and Lemma 4.5, we obtain

Proposition 4.6. Under the assumptions of Theorem 1.1, there exists a symplectic embedding $F:(K, \omega) \rightarrow\left(\mathbf{C}^{n}, \omega_{0}\right)$ (depending on $K$ ) for every compact subset $K \subset X$.

Proof of Theorem 1.1. It follows from Proposition 4.2 that $X$ is a Stein manifold. From Proposition 4.6, we know that for every energy value $c>0$ we can pick a number $c^{\prime}>c$ so that there exists a symplectic embedding $F:\left(K^{\prime}, \omega\right) \rightarrow\left(\mathbf{C}^{n}, \omega_{0}\right)$ for the compact subset $K^{\prime}=\left\{f \leq c^{\prime}\right\} \subset X$. Since every compact subset of $\mathbf{C}^{n}$ has finite capacity, the monotonicity property of the capacity function $c_{0}$ implies that $K^{\prime}$ has finite capacity $c_{0}\left(K^{\prime}, \omega\right)$ hence Theorem 3.2 applies. This completes the proof of Theorem 1.1.

\section{References.}

[1] H.-D. Cao, Deformation of Kähler metrics to Kähler -Einstein metrics on compact Kähler manifolds, Invent. Math. 81 (1985), 359-372.

[2] H.-D. Cao, On Hanarck's inequalities for the Kähler-Ricci flow, Invent. Math. 109 (1992), 247-263.

[3] H.-D. Cao, Existence of gradient Kähler-Ricci solitons, Elliptic and Parabolic Methods in Geometry, (Minneapolis, MN, 1994) B. Chow, R. Gulliver, S. Levy, J. Sullivan ed., AK Peters (1996), 1-16.

[4] H.-D. Cao, Limits of solutions to the Kähler-Ricci flow, J. Differ. Geom., 45 (1997), 257-272.

[5] Y. Eliashberg, M. Gromov, Convex symplectic manifolds, in Proc. Symp. Pure Math. 52 (1991), Part 2, 135-162.

[6] R. S. Hamilton, The Ricci flow on surfaces, in Mathematics and General Relativity, Contemporary Mathematics 71 (1986).

[7] R. S. Hamilton, The Hanarck estimate for the Ricci flow, J. Differ. Geom. 37 (1993), 225-243. 
[8] R. S. Hamilton, Eternal solutions to the Ricci flow, J. Differ. Geom. 38 (1993), $1-11$.

[9] R. S. Hamilton, An isoperimetric estimate for the Ricci flow on the 2-sphere, Modern methods in complex analysis (Princeton, NJ, 1992), 201-222, Ann. of Math. Stud., 137, Princeton Univ. Press, 1995.

[10] R. S. Hamilton, Formation of singularities in the Ricci flow, Surveys in Diff. Geom. 2 (1995), 7-136, International Press, Boston.

[11] H. Hofer, E. Zehnder, Symplectic invariants and Hamiltonian dynamics, Birkhäuser, 1994.

[12] N. Mok, The uniformization theorem for compact Kähler manifolds of nonnegative holomorphic bisectional curvature, J. Differ. Geom. 27 (1988), 179-214.

TeXas A\&M University

College Station

E-mail address: cao@math.tamu.edu

AND

UNIVERSITY OF CALIFORNIA

LA Jolla

AND

Columbia University

NEW YORK

Received February 18, 1998. 